\title{
Calcifying Pseudoneoplasms of the Skull Base Presenting with Cranial Neuropathies: Case Report and Literature Review
}

\author{
Yoichi Nonaka ${ }^{1}$ Hamid R. Aliabadi ${ }^{1}$ Allan H. Friedman ${ }^{1}$ Fred G. Odere ${ }^{2}$ Takanori Fukushima ${ }^{1,3}$ \\ ${ }^{1}$ Division of Neurosurgery, Duke University Medical Center, Durham, \\ North Carolina, United States \\ 2 Division of Pathology, Duke University Medical Center, Durham, \\ North Carolina, United States

\begin{abstract}
Address for correspondence and reprint requests Yoichi Nonaka, M.D., Division of Neurosurgery, Duke University Medical Center, Box 3807, 1000 Trent Drive 4520 Hosp South, Durham, NC,
\end{abstract} \\ United States, 27710 (e-mail: ynonaka1971@yahoo.co.jp).
}

${ }^{3}$ Carolina Neuroscience Institute, Raleigh, North Carolina, United States

J Neurol Surg Rep 2012;73:41-47.

\begin{abstract}
Keywords

- brain stone

- calcifying pseudoneoplasm

- cerebral calculi

- fibro-osseous lesion

- neural axis
\end{abstract}

\section{Introduction}

Fibro-osseous lesions, also reported as cerebral calculi, brain stones, or calcifying pseudoneoplasms of the neuraxis are extremely rare. These lesions demonstrate a characteristic histopathologic appearance. Since the first report by Miller in $1922,{ }^{1} 29$ articles (56 cases) have been reported in the international literature. ${ }^{1-29}$ These lesions appear to be slow growing and usually carry an excellent prognosis with gross total excision.

The pathogenesis of these lesions remains unclear, but a reactive proliferative process, rather than a neoplastic one, is favored by most investigators. ${ }^{2-7}$ In addition, it is also believed that this lesion is probably an unusual expression of tumoral calcinosis. They can occur anywhere within the neuraxis, both intra-axial and extra-axial. The patients with intracranial lesion often presented with seizure, headache, or other symptoms related to local compression, whereas patients with spinal lesion commonly presented with pain in the affected area. In this report we describe our experience of calcifying pseudoneoplasms, one located in the infratemporal fossa adjacent to the foramen rotundum, and the other in the posterior fossa involving one of the occipital condyles (previously reported), both of which caused cranial nerve deficits. ${ }^{8}$ The appearance of this unusual tumor is illustrated, the radiological evaluation and pathological findings are discussed, and the literature is reviewed. received

April 14, 2011

accepted after revision

January 14, 2012

published online

July 2, 2012
Copyright $\odot 2012$ by Thieme Medical Publishers, Inc., 333 Seventh Avenue, New York, NY 10001, USA. Tel: +1(212) 584-4662.
DOI http://dx.doi.org/ 10.1055/s-0032-1321503. ISSN 2193-6358. 


\section{Case Reports}

\section{Case 1}

A 56-year-old man with a history of chronic right-ear infections had images ordered by his primary care physician for evaluation. A computed tomography (CT) scan and subsequent magnetic resonance imaging (MRI) scan were performed. These images showed a partially calcified mass in the anterior infratemporal fossa on the right side. The patient was also having right-sided facial numbness and occasional orbital pain around the maxillary branch of the trigeminal nerve (V2). He was seen and evaluated by an otolaryngologist, who attempted a transnasal approach to the lesion. However, due to technical difficulties in gaining complete access to the lesion, the surgery was unsuccessful. The patient was referred to our institution to be considered for a skull base approach to excise the lesion.

\section{Examination}

A CT scan revealed a partially calcified mass lesion located inferior to the floor of the right anterior temporal fossa (-Fig. 1A). MRI demonstrated a mass that was heterogeneous in signal ( - Figs. 1B, C, D). The mass measured $2.0 \times 1.9 \mathrm{~cm}$ in greatest dimensions. It was largely hyperintense on the T2-weighted image, with multiple small-lobulated areas of hypointensity within. There were some areas of mild heterogeneous enhancement within the lesion. The mass abutted the floor of the posterior aspect of the right orbit.

\section{Operation}

A right preauricular anterior infratemporal fossa skull base approach was performed. After myocutaneous flap elevation, the anterior temporal region was well exposed. A subtemporal craniotomy was made. The V2 nerve was exposed easily at the markedly enlarged foramen rotundum with the extradural temporopolar approach. The mass was identified under the V2 nerve. The tumor was gradually elevated from the surrounding tissues with careful attention to the abducens nerve. It was a very smooth, rounded mass with a large roundish central core of calcification. Postoperative course was uneventful. His facial numbness still remained slightly, but he denied any orbital pain. Postoperative MRI demonstrated no obvious residual tumor ( $\mathbf{- F i g . ~ 1 E ) . ~}$

\section{Pathological Examination}

Multiple pinkish-white tissue fragments and bone fragments were noted within the lesion, with an aggregate dimension of $3.5 \times 3.0 \times 1.0 \mathrm{~cm}$. Microscopic examination showed a sparsely cellular fibromyxoid stroma containing bland fibroblastic nuclei and scattered blood vessels. It also showed a large nerve with associated ganglion cells involved by hypocellular fibrillar tissue that is partly calcified ( $\mathbf{- F i g . ~ 2 A ) . ~ T h e r e ~}$ was no evidence of neoplasia, malignancy, or acute inflammation. There was no epidermal inclusion cyst or squamous epithelium, nor was there keratin. Immunohistochemical analysis was not performed in this case. The final histological diagnosis was consistent with a calcifying pseudoneoplasm.
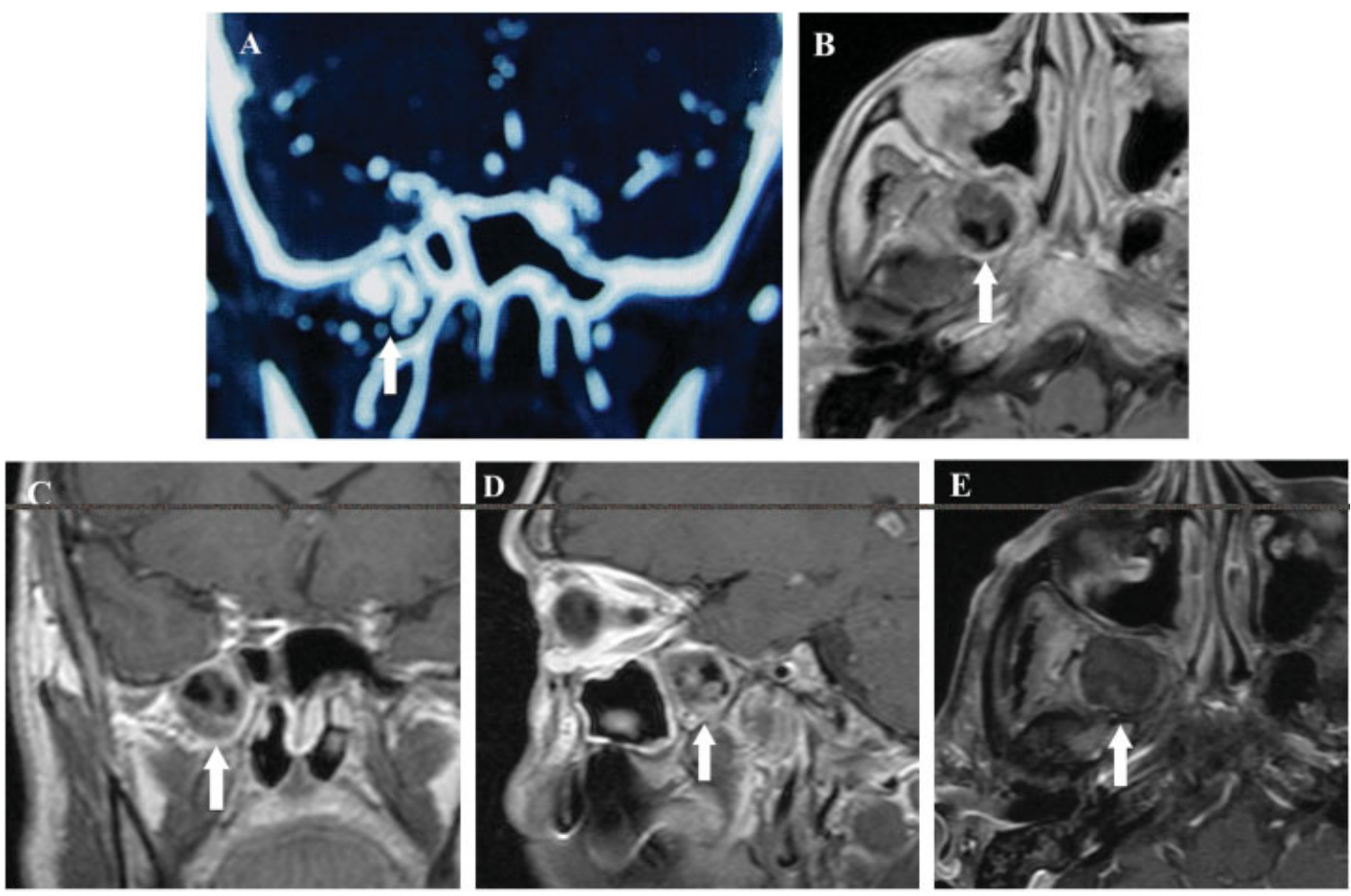

Figure 1 Pre- and post-operative magnetic resonance imaging (MRI) and computed tomography (CT) images (Case 1) Preoperative coronal $\mathrm{CT}$ scan demonstrated a mass with calcification in the right infratemporal fossa (A). Preoperative axial, coronal, and sagittal postcontrast MRIs (B, C, D) demonstrated a mass in the right infratemporal fossa. The mass was heterogenous in signal. Postoperative MRI demonstrated no obvious residual tumor (E). 

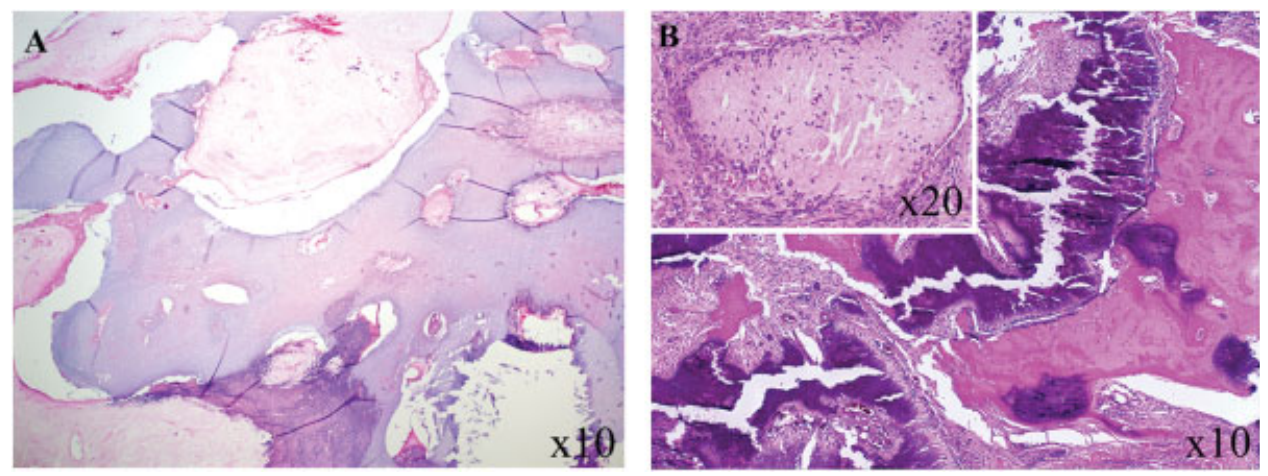

Figure 2 Photomicrograph microscopic examination of Case 1 showed a sparsely cellular fibromyxoid stroma containing bland fibroblastic nuclei, scattered blood vessels, and a large nerve with associated ganglion cells involved by hypocellular fibrillar tissue with extensive calcification that is amorphous (A). No epithelial component is seen. Hematoxylin and eosin ( $\mathrm{H} \& \mathrm{E})$, original magnification x10. Photomicrograph of surgical specimens obtained in Case 2 demonstrating calcified tissue containing amorphous granular and fibrillar material (B). There are focal ossification and cells that resemble meningothelial cells ( $\mathrm{H} \& \mathrm{E}$, original magnification $\times 10$ ). A higher magnified image at $x 20$ (left upper) showing spindle cells on the perimeter.

\section{Case 2}

A 35-year-old man presented with a 6-month history of progressively worsening occipital headaches with some generalized fatigue, tinnitus, and dizziness.

\section{Examination}

Initial neurological examination showed a slight asymmetry of the soft palate and the tongue deviation toward the left side. Neither obvious tongue atrophy nor any fasciculations were noted. He had a subtle left dysmetria and a negative Romberg's sign. He eventually underwent CT and MRI scans. The CT scan demonstrated a large calcified mass in the left posterior fossa that appeared to arise from the left occipital condyle, causing mass effect onto the brainstem and cerebellum. MRI imaging demonstrated a $4.3 \times 2.9 \times 2.9 \mathrm{~cm}$ mass that involved the left posterior aspect of the clivus and extended exophytically into the posterior fossa (-Fig. 3A, B). A majority of the mass projected intradurally. There was rightward deviation of the brainstem and narrowing of the inferior aspect of the fourth ventricle.

\section{Operation}

A left far-lateral transcondylar approach was performed along with a retro-infralabyrinthine mastoidectomy. During the partial mastoidectomy, the left sigmoid sinus was found to be occluded. The mastoidectomy was followed anteriorly until the facial nerve was identified. The lateral suboccipital craniotomy was then performed and the foramen magnum was opened. The tumor had eroded through the bone into the foramen magnum. The condyle then was partially resected. The condyle was filled with tumor, which was mostly calcified; the presence of large balls of calcium made us think that this might have been a meningioma. The dura was involved with the tumor. Underneath, a hard bony mass was found,

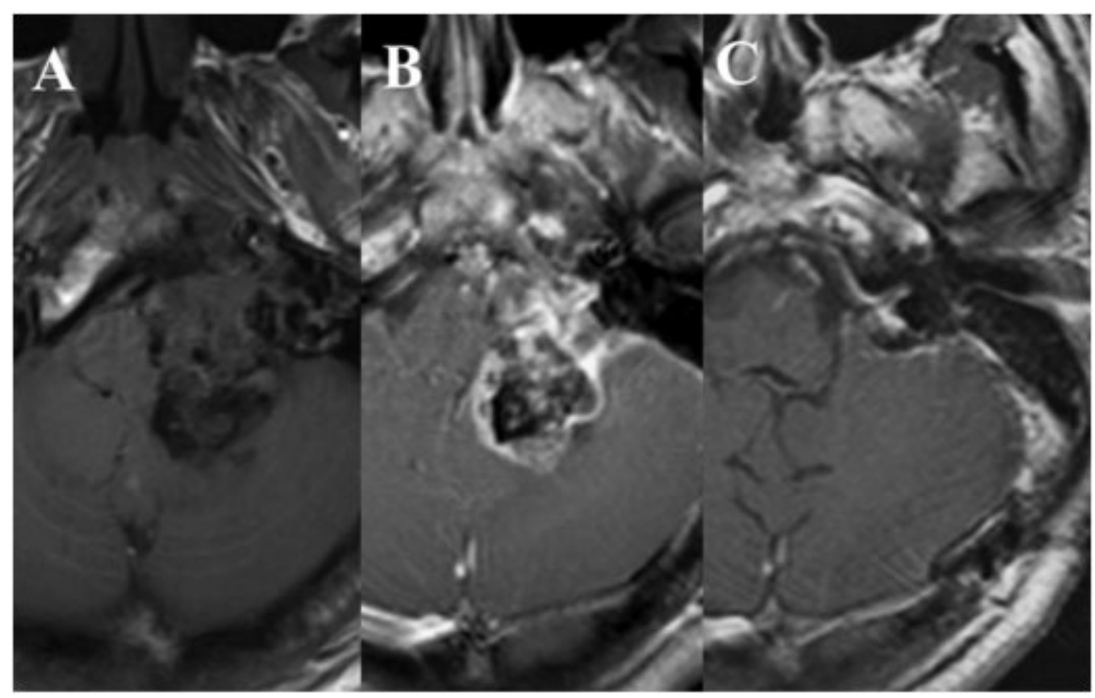

Figure 3 Pre- and post-operative axial magnetic resonance (MR) images (Case 2). Preoperative axial pre- and post-contrast T1-weighted MR images showing a mass compression to the brainstem with heterogeneous rim enhancement (A, B). Postoperative postcontrast T1-weighted MR image shows the removal of the mass (C). 
which was removed in a piecemeal fashion. Some of the softer calcified areas were removed using an ultrasonic aspirator. The eleventh cranial nerve (CN11) was found to be adherent to the tumor. This was separated. The degenerated cerebellar tonsil was covering a good portion of the tumor, though we would not have known this without actually exposing the area. A small capsular portion of tumor adherent to the posterior inferior cerebellar artery (PICA) and to the lower cranial nerves ( $\mathrm{CNs}$ ) (IX and $\mathrm{X}$ ) was left to avoid damage to the $\mathrm{CNs}$ and to the brainstem. The tumor was resected in a subtotal fashion. Postoperative course was uneventful. Postoperative neurological examination was normal except for the tongue deviation toward the left side, which was stable from preoperative examination. Postoperative 6-month MRI demonstrated only a small remnant of capsule (-Fig. 3-C).

\section{Pathological Examination}

Microscopic examination demonstrated calcified tissue containing amorphous granular and fibrillar material (-Fig. 2B). There were focal ossification, cells that resemble meningothelial cells, and palisading spindle cells around the myxoid amorphous calcifying nodules. Hemosiderin and chronic inflammation were seen. Tumor cells did not show immunoreactivity for epithelial membrane antigen (EMA), neuron-specific enolase (NSE), and S-100 protein. The final pathological diagnosis was consistent with a calcifying pseudoneoplasm.

\section{Discussion}

Calcified, fibro-osseous lesions known as calcifying pseudoneoplasms of the neuraxis, cerebral calculi, or brain stones are extremely rare and are usually seen at the cranial base, commonly adjacent to the dura mater or the arachnoid. ${ }^{1-29}$ However, they can occur anywhere within the neuraxis. ${ }^{2,3,5}$ It has been suggested, but not proven, that calcifying pseudoneoplasms may develop as a healing response to an array of inciting factors, which can account for the variations in histopathologic features. ${ }^{3,5,7}$ Although the histopathologic characteristics of this unusual lesion are not yet understood, a reactive rather than a hamartomatous process has been favored. ${ }^{3,5}$

Our review of the literature revealed 29 reported articles with 56 cases of these lesions (-Table 1). Articles were identified via PubMed search using the key words "fibroosseous lesion," “calcifying pseudoneoplasm," "pseudotumor," "cerebral calculi," "brain stone," "neural axis," "neuraxis," "central nervous system (CNS)," "intracranial," "brain," and "spine" alone and in combination.

The first reported case was described as "calculi within the brain" by Miller in $1922 .{ }^{1}$ The terms "calcifying pseudoneoplasms of the neural axis" was used by Bertoni et al at first in 1990. There have been 30 male and 23 female patients (gender unknown in three cases) reported, with ages ranging from 6 to 83 years (mean age 46.1 years). These lesions most often arose from intracranial space (intracranial type: 37 cases; 66.1\%) followed by spine (spine type: 15 cases; 26.8\%) and craniovertebral junction (CVJ type: four cases;
7.1\%). The mean age of intracranial type, CVJ type, and spine type were $44.6,52.8$, and 47.7 year respectively.

The patients with intracranial type most commonly presented with seizure (31.6\%) followed by headache (13.2\%) and cerebellar sign (5.3\%). ${ }^{1-23}$ Three patients $(8.1 \%)$ were found incidentally, and seven cases (18.9\%) were autopsy findings. Only four cases out of 37 presented with symptoms related to $\mathrm{CN}$ involvement. ${ }^{2,3,17}$ Over half of the intracranial type lesions were seen in the supratentorial region (54.1\%). Infratentorial lesions were seen in $29.7 \%$ of them (not described in six cases). The patients with CVJ type most commonly presented with neck pain (75\%), followed by headache (25\%), facial pain (25\%) and lower-extremity stiffness. ${ }^{3,5,20,24}$ Three of four cases were mainly located at the foramen magnum and another at the clivus to the upper cervical spine.

The patients with the "spinal" type commonly presented with pain in the affected area (86.7\%: back pain; seven cases, neck pain; four cases, hip pain; two cases; and leg pain, one case) and gait disturbance (20.0\%) or sensorial disturbance $(13.3 \%) .^{3,5,25-29}$ Only one case (6.6\%) was found incidentally. The lesions were seen at the cervical spine (46.7\%), lumber spine (33.3\%), and thoracic spine (20\%). Among them, C2, $\mathrm{T}-10$, and L4-L5 were the most commonly affected levels. Interestingly, $86.7 \%$ of the spine type lesions were found at the extradural location near the intervertebral disc level (one case in the subarachnoid space), whereas all cases except for one (97.7\%; not described in one case) of intracranial type and all of CVJ-type lesions occurred in the intradural space. Only two cases out of 57 reported cases had suffered a recurrence a couple of years after the initial excision, however, the outcome is often considered to be excellent even if the tumor was not resected completely. ${ }^{3,25}$

When considering the differential diagnosis for calcifying intra-axial or extra-axial lesions, the uniform T1 and T2 hypointensity without solid enhancement is a key distinguishing feature. ${ }^{2}$ The primary differential considerations for the extra-axial calcifying pseudoneoplasms at the skull base are meningioma, chordoma, chondrosarcoma, and vestibular schwannoma. Specifically, the uniform T2 hypointensity without enhancement would be unusual for chordoma, chondrosarcoma, or vestibular schwannoma. Important differential diagnosis considerations for intra-axial calcified masses include such tumors as gangliogliomas and oligodendrogliomas, vascular lesions such as cavernous malformations, and even infections such as tuberculosis. ${ }^{2,3}$ The differential diagnosis for spine lesions included disc herniation, synovial cyst, epidural abscess, meningioma, or possibly an old calcified hematoma. ${ }^{26}$

Calcifying pseudoneoplasms are composed of fibro-osseous tissue with an amorphous calcification mixed with spindle and giant cells palisading generally around the chondroid or calcifying material. ${ }^{2-7}$ Microscopically, the differential diagnosis of calcifying pseudoneoplasms includes a variety of reactive lesions such as granulomatous disease, herniated disk material, and even neoplastic lesions like chondroid chordoma, chondroma, chondrosarcoma, metaplastic meningioma, or calcified primary parenchymal central nervous system tumors. ${ }^{6}$ In most cases, immunohistochemistry findings for 


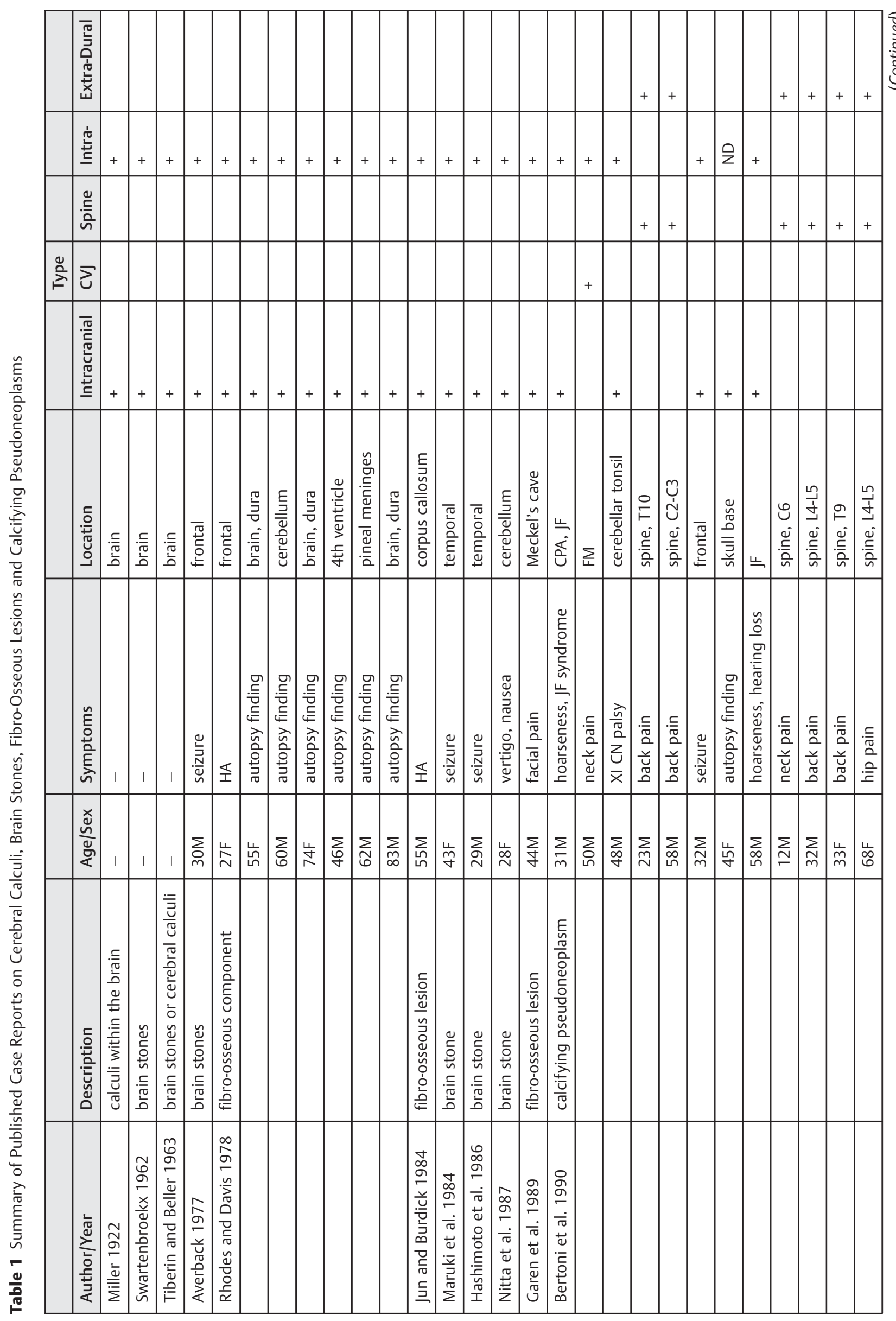




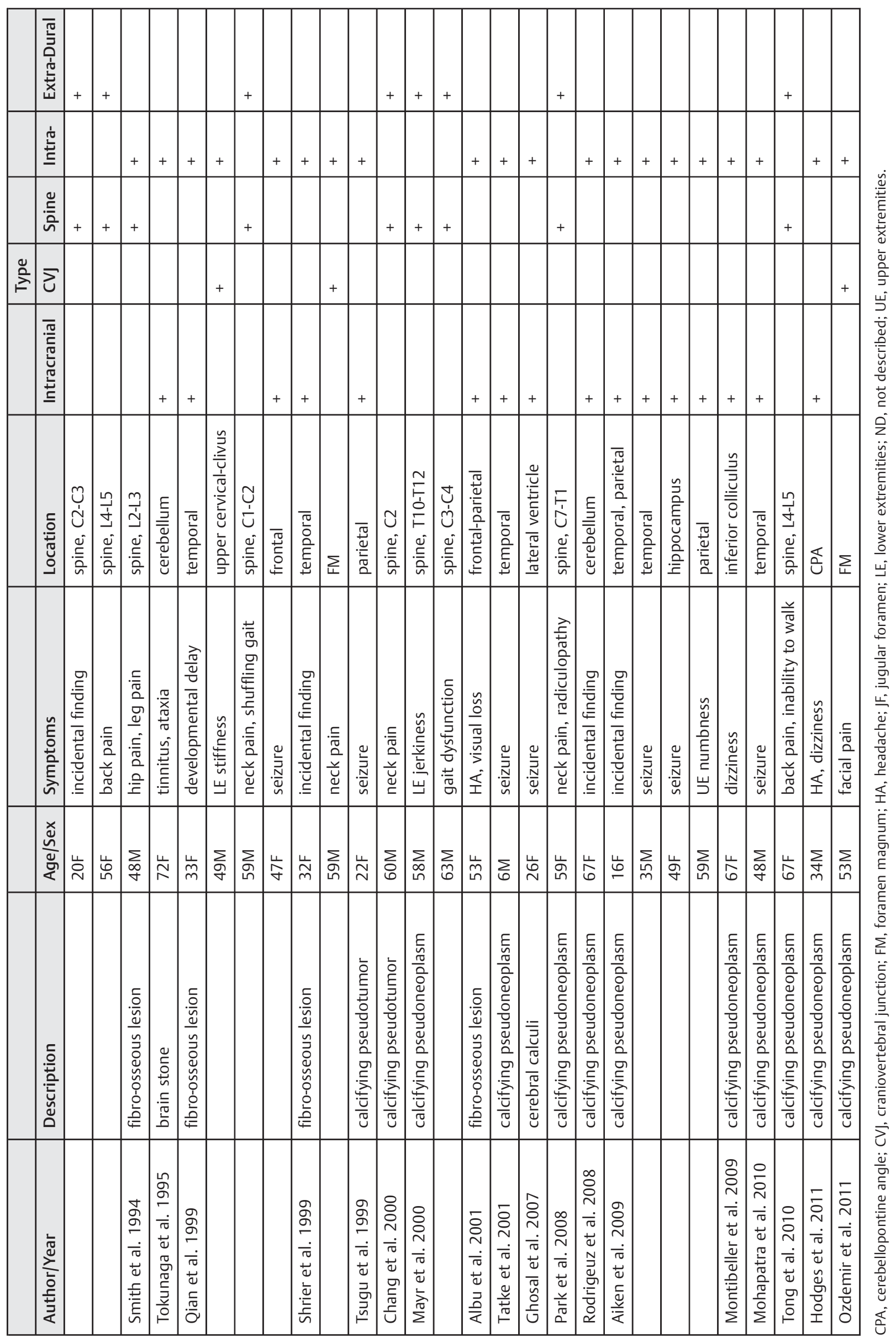


vimentin were positive, with negative staining for glial fibrillary acidic protein and S-100., 3,6,17,19 Vimentin immunoreactivity was noted in peripheral palisading cells, but the intensity varied from case to case. ${ }^{5,17}$ EMA was sometimes positive for the spindle, stromal, and epithelioid cells surrounding the matrix ${ }^{2,5,6}$ and was negative in some other cases. ${ }^{4,7,19}$ However, unfortunately, immunohistochemical findings have not contributed significantly in determining the histogenesis of calcifying pseudoneoplasms. ${ }^{27}$

The classic histopathologic features in calcifying pseudoneoplasms include a distinctive set of common elements: (1) typical chondromyxoid matrix in a nodular pattern; (2) palisading spindle to epithelioid cells; (3) variable amounts of fibrous stroma, (4) calcification, osseous metaplasia, and scattered psammoma bodies; and (5) foreign-body reaction with giant cells. ${ }^{5}$ However, not all classic histopathologic signs are present in all calcifying pseudoneoplasms. The presence of each component is highly variable in individual lesions. In our reported cases, the pathological examination (second case) demonstrated the typical characteristics of a calcifying pseudoneoplasm; however, the first case we reported showed only some of these features.

\section{Conclusion}

Calcifying pseudoneoplasms appear to be slow-growing lesions that tend to present secondary to local compression or irritation of adjacent tissues. Cases with spinal lesion presented with pain, gait disturbance, radiculopathy, or myelopathy related to the affected area, and other intracranial lesions presented with seizure, headache, and cerebellar signs caused by tumor compression. Our two cases demonstrated apparent $\mathrm{CN}$ deficits with encasement or involvement with tumor.

Although calcifying pseudoneoplasms are histopathologically benign, surgical outcome depends on the tumor location, tumor extension, and the degree of adhesion. Surgical technique to preserve the involved $\mathrm{CNs}$ and vasculature is essential. Given that the prognosis is favorable even with subtotal resection, we do not recommend performing an extensive or morbid surgical procedure. It may be sufficient to debulk the tumor just enough to decompress the neural elements.

\section{References}

1 Miller EA. Calculi within the brain. Report of a case of intracranial calcification with successful operation and recovery. Surg Gynecol Obstet 1922;34:786-789

2 Aiken AH, Akgun H, Tihan T, Barbaro N, Glastonbury C. Calcifying pseudoneoplasms of the neuraxis: CT, MR imaging, and histologic features. AJNR Am J Neuroradiol 2009;30:1256-1260

3 Bertoni F, Unni KK, Dahlin DC, Beabout JW, Onofrio BM. Calcifying pseudoneoplasms of the neural axis. J Neurosurg 1990; 72:42-48

4 Montibeller GR, Stan AC, Krauss JK, Nakamura M. Calcifying pseudoneoplasm of the inferior colliculus: an unusual location for a rare tumor: case report. Neurosurgery 2009;65: E1005-E1006, discussion E1006
5 Qian J, Rubio A, Powers JM, et al. Fibro-osseous lesions of the central nervous system: report of four cases and literature review. Am J Surg Pathol 1999;23:1270-1275

6 Rodriguez FJ, Scheithauer BW, Fourney DR, Robinson CA. Ependymoma and intraparenchymal calcifying pseudoneoplasm of the neural axis: incidental collision or unique reactive phenomenon? Acta Neuropathol 2008;115:363-366

7 Tatke M, Singh AK, Gupta V. Calcifying pseudoneoplasm of the CNS. Br J Neurosurg 2001;15:521-523

8 Hodges TR, Karikari IO, Nimjee SM, et al. Calcifying pseudoneoplasm of the cerebellopontine angle: case report. Neurosurgery 2011;69(1 suppl operative):onsE117-E120

9 Rhodes RH, Davis RL. An unusual fibro-osseous component in intracranial lesions. Hum Pathol 1978;9:309-319

10 Swartenbroekx A. [A case of "brain stones"]. [in Dutch] J Belge Radiol 1962;45:534-535

11 Tiberin P, Beller AJ. Observations on so-called brain stones or cerebral calculi. Neurology 1963;13:464-476

12 Averback P. Epileptogenic mineralization: pathological variants with good prognosis. Ann Neurol 1977;2:332-335

13 Maruki C, Nakajima K, Shimoji T, Ito K, Matsumoto M, Ishii S. [Brain stone. A case report]. [in Japanese] No Shinkei Geka 1984; 12:1441-1445

14 Hashimoto M, Tanaka T, Ohgami S, Yonemasu Y, Fujita M. [A case of idiopathic brain stone presenting as psychomotor epilepsy]. [in Japanese] No Shinkei Geka 1986;14:1457-1461

15 Nitta T, Ito M, Sato K, Ishii S. [Brain stone in the cerebellum. Case report]. [in Japanese] Neurol Med Chir (Tokyo) 1987;27:150-153

16 Tokunaga $\mathrm{H}$, Iwanaga $\mathrm{H}$, Imanishi M, et al. [A huge idiopathic brain stone in the posterior fossa]. [in Japanese] No Shinkei Geka 1995;23:711-716

17 Garen PD, Powers JM, King JS, Perot PL Jr. Intracranial fibroosseous lesion. Case report. J Neurosurg 1989;70:475-477

18 Jun C, Burdick B. An unusual fibro-osseous lesion of the brain. Case report. J Neurosurg 1984;60:1308-1311

19 Tsugu H, Fukushima T, Takeno Y. Calcifying pseudotumor of the neural axis-case report. Neurol Med Chir (Tokyo) 1999;39: 762-765

20 Shrier DA, Melville D, Millet D, et al. Fibro-osseous lesions involving the brain: MRI. Neuroradiology 1999;41:18-21

21 Albu G, Deák G, Mencser Z, Vajtai I. [Fibro-osseous lesion of the central nervous system]. Orv Hetil 2001;142:1165-1167

22 Ghosal N, Thakre D, Murthy G, Hegde AS. Cerebral calculi in the temporal horn of the lateral ventricle: report of an unusual case. Histopathology 2007;50:817-818

23 Mohapatra I, Manish R, Mahadevan A, Prasad C, Sampath S, Shankar SK. Calcifying pseudoneoplasm (fibro osseous lesion) of neuraxis (CAPNON) - a case report. Clin Neuropathol 2010;29: 223-226

24 Ozdemir M, Bozkurt M, Ozgural O, Erden E, Tuna H, Caglar YS. Unusual localization of an unusual tumor: calcifying pseudoneoplasm of the foramen magnum. Clin Neuropathol 2011;30:25-27

25 Chang H, Park JB, Kim KW. Intraosseous calcifying pseudotumor of the axis: a case report. Spine (Phila Pa 1976) 2000;25:1036-1139

26 Mayr MT, Hunter S, Erwood SC, Haid RW Jr. Calcifying pseudoneoplasms of the spine with myelopathy. Report of two cases. J Neurosurg 2000;93(2, Suppl):291-293

27 Park P, Schmidt LA, Shah GV, Tran NK, Gandhi D, La Marca F. Calcifying pseudoneoplasm of the spine. Clin Neurol Neurosurg 2008;110:392-395

28 Smith DM, Berry AD III. Unusual fibro-osseous lesion of the spinal cord with positive staining for glial fibrillary acidic protein and radiological progression: a case report. Hum Pathol 1994;25: 835-838

29 Tong D, Karunaratne N, Howe G, Spencer D, Manolios N. Clinical images: Calcifying pseudoneoplasm of the neuraxis. Arthritis Rheum 2010;62:704 